\title{
Recurrent Clostridioides difficile infection: Recognition, management, prevention
}

\section{ABSTRACT}

Clostridioides difficile infection (CDI) is the most common cause of diarrhea in hospitalized patients and results in substantial morbidity, mortality, and costs. Its clinical management, primarily with antibiotics, is often complicated by recurrent episodes. These recurrent $\mathrm{CDI}$ episodes are thought to be caused by antibiotic disruption of colonic microbiota and usually occur within 4 weeks of completing antibiotic therapy. The risk of recurrent $C D I$ increases after the first episode, creating a need for management strategies to diagnose, treat, and prevent these complications.

\section{KEY POINTS}

Diagnostic testing for CDI should be performed only in symptomatic patients.

Diagnosis is based on unexplained diarrhea and a positive $C$ difficile assay.

The goal of therapy for recurrent $C D I$ is to allow the normal colonic microbiota to restore itself.

Fecal microbiota transplantation has shown efficacy for treating recurrent CDI.

Antimicrobial stewardship and infection prevention are key strategies for preventing $C D I$.

Medical Grand Rounds articles are based on edited transcripts from Medicine Grand Rounds presentations at Cleveland Clinic. They are approved by the author but are not peer-reviewed.

doi:10.3949/ccjm.87gr.20001

\begin{abstract}
Clostridioides (formerly Clostridium) difficile) is an anaerobic spore-forming bacillus that colonizes the intestinal tract in patients whose normal gut microbiota is disrupted by antibiotic therapy. ${ }^{1} \mathrm{C}$ difficile produces 2 major toxins-toxins $\mathrm{A}$ and $\mathrm{B}$ - that cause intestinal mucosal injury, diarrhea, and colitis, and in some cases, fulminant infection leading to shock, ileus, and toxic megacolon. ${ }^{2} \mathrm{C}$ difficile infection (CDI) recurs in up to one-quarter or more of treated patients, complicating its management.

In the United States, $\mathrm{C}$ difficile is a common hospital-acquired infection, affecting about 500,000 patients annually, causing up to 30,000 deaths, and incurring inpatient costs of nearly $\$ 5$ billion. $^{2-4}$ This article reviews the current standards for diagnosing and treating $\mathrm{CDI}$ and discusses strategies for managing and preventing recurrent disease.
\end{abstract}

\section{DIAGNOSIS}

The current standard for diagnosis of CDI requires both unexplained new diarrhea and a positive result on a $C$ difficile assay. ${ }^{2,5}$ Guidelines recommend laboratory testing for $\mathrm{C}$ difficile only in patients who have symptoms, defined as unexplained new onset of 3 or more unformed stools per day. Also, practitioners need to rule out use of a laxative (eg, polyethylene glycol) in the preceding 48 hours or a history of chronic diarrhea with no change in symptoms. Table 1 lists laboratory assays for detecting $\mathrm{C}$ difficile toxin or organism. ${ }^{2,5}$

Colonization vs infection: Is it important?

$\mathrm{C}$ difficile colonization is the existence of the organism or toxin in the stool of patients who do not have unexplained new diarrhea. $\mathrm{C}$ difficile infection is the existence of the organism

\section{Diagnosis \\ requires \\ unexplained \\ new-onset \\ diarrhea \\ and a positive \\ C difficile assay}




\section{TABLE 1}

\section{Diagnostic tests for Clostridioides difficile}

Test

\section{Organism detection assays}

Nucleic acid amplification tests

(eg, polymerase chain reaction)

Glutamate dehydrogenase

Toxigenic $C$ difficile culture
Characteristics

Sensitivity, specificity
Detects toxin gene (ie, organism)

but not toxins

C difficile common antigen

Growth of $C$ difficile organism

Testing not readily available

Slow turnaround time
High sensitivity

Low to moderate specificity

High sensitivity

Low specificity

High sensitivity

Low specificity

\section{Toxin detection assays}

Enzyme immunoassay

Cell culture cytotoxicity neutralization assay
Detects free toxins

Detects free toxins

Lacks standardization

Slow turnaround time
Low sensitivity Moderate specificity

High sensitivity

High specificity, if optimized

Information from McDonald et al, reference 2

Laboratory testing cannot distinguish between asymptomatic C difficile colonization

and

symptomatic CDI or toxin in patients with unexplained new diarrhea. Laboratory testing cannot distinguish between asymptomatic $\mathrm{C}$ difficile colonization and symptomatic CDI.

The prevalence of asymptomatic C difficile stool colonization varies from $3 \%$ to $26 \%$ in adult hospitalized patients to $5 \%$ to $7 \%$ in elderly patients in long-term care facilities. In asymptomatic adults without any recent healthcare exposure, the prevalence is less than $2 \% .^{2}$

A patient presenting with no diarrhea, a positive polymerase chain reaction (PCR) test, and a negative enzyme immunoassay (EIA) test likely has $\mathrm{C}$ difficile colonization. However, a patient can have a positive EIA result without symptoms, so the best approach is to carefully assess the patient for unexplained new-onset diarrhea.

Institutional policies will determine which CDI tests are used. If the policy is to test only stool specimens from patients with unexplained and new onset of at least 3 unformed stools in 24 hours, one has the following options ${ }^{2}$ :
- Order a nucleic acid amplification test (NAAT) alone (eg, PCR)

or

- Order a stool toxin test as part of a multistep algorithm:

1) NAAT plus toxin test, or

2) Glutamate dehydrogenase $(\mathrm{GDH})$ plus toxin test, or

3) GDH plus toxin test, arbitrated by NAAT.

If the facility does not have a policy to submit stool specimens for CDI testing only from patients who have unexplained new-onset diarrhea, a NAAT (eg, PCR) test alone is not recommended because it increases the chance of detecting colonization. Instead, the recommendation is to perform a stool toxin test as part of a multistep algorithm:

1) NAAT plus toxin test, or

2) GDH plus toxin test, or

3) $\mathrm{GDH}$ plus toxin test, arbitrated by NAAT

In addition, repeat $C$ difficile testing is not recommended to evaluate for cure in patients whose symptoms have improved or resolved. 
That is because $\mathrm{C}$ difficile can continue to be shed in stools for more than 1 month, even after a patient's symptoms have resolved. ${ }^{6}$ This is important because treatment of asymptomatic $\mathrm{C}$ difficile carriers with either metronidazole or vancomycin has not been shown to be beneficial, and vancomycin can prolong $\mathrm{C}$ difficile colonization or increase the risk of acquiring a new $\mathrm{C}$ difficile strain. ${ }^{?}$

\section{TREATMENT OF A FIRST EPISODE}

The first step in treating CDI is to stop the inciting antibiotic therapy as soon as possible. Antiperistaltic therapy should be avoided, especially if the patient is not receiving antibiotics for CDI. ${ }^{2,8}$ Additionally, empiric anti-C difficile therapy is not recommended unless a substantial delay in $\mathrm{C}$ difficile testing results is anticipated or the patient has fulminant CDI.

Treatment of initial episodes of CDI, as outlined in clinical practice guidelines from the Infectious Diseases Society of America (IDSA) and Society for Healthcare Epidemiology of America (SHEA), ${ }^{2}$ is based on the severity of disease.

Nonsevere cases are defined as those in which the white blood cell count remains less than or equal to $15.0 \times 10^{9} / \mathrm{L}$ and the serum creatinine level is less than $1.5 \mathrm{mg} / \mathrm{dL}$. In such cases, there are 3 options for treatment:

- Vancomycin $125 \mathrm{mg}$ by mouth 4 times a day for 10 days

- Fidaxomicin $200 \mathrm{mg}$ by mouth twice a day for 10 days (more about this agent below)

- Metronidazole $500 \mathrm{mg}$ by mouth 3 times a day for 10 days (if access to vancomycin or fidaxomicin is limited).

The first 2 options carry strong recommendations based on high levels of evidence, whereas the third has a weak recommendation based on a high level of evidence. ${ }^{2}$

Severe cases are those in which the white blood cell count is $15.0 \times 10^{9} / \mathrm{L}$ or higher or the serum creatinine level is higher than 1.5 $\mathrm{mg} / \mathrm{dL}$. There are 2 treatment options:

- Vancomycin $125 \mathrm{mg}$ by mouth 4 times a day for 10 days

- Fidaxomicin $200 \mathrm{mg}$ by mouth twice a day for 10 days.

These options carry strong recommendations based on high levels of evidence. ${ }^{2}$
Fulminant cases are characterized by hypotension, shock, ileus, or toxic megacolon. Treatment is with vancomycin $500 \mathrm{mg}$ by mouth or nasogastric tube 4 times a day, plus metronidazole $500 \mathrm{mg}$ intravenously every 8 hours, especially if the patient has ileus. In addition, if the patient has ileus, one can consider rectal installation of vancomycin 500 mg. ${ }^{2}$

Oral vancomycin therapy for fulminant CDI carries a strong recommendation based on a moderate level of evidence; intravenous metronidazole carries a strong recommendation based on a moderate level of evidence, and rectal vancomycin carries a weak recommendation based on a low level of evidence. ${ }^{2}$

With respect to other therapies for fulminant CDI, there are limited data regarding the use of fidaxomicin. Patients with life-threatening or fulminant CDI or toxic megacolon were excluded from clinical trials evaluating fidaxomicin. ${ }^{9,10}$ Additionally, there are limited data on the use of fecal microbiota transplantation (FMT) for fulminant CDI. ${ }^{2}$

\section{RECURRENT CDI}

A major clinical challenge is recurrent CDI, which usually occurs within 4 weeks after completion of anti-C difficile therapy. The risk of recurrence increases with each episode ${ }^{11,12}$ :

- Up to $20 \%$ to $25 \%$ after the first CDI episode

- Up to $40 \%$ to $45 \%$ after the second CDI episode

- More than $60 \%$ to $65 \%$ after 3 or more CDI episodes.

It is recommended that patients with CDI be counseled regarding the risk of recurrence. If a patient's diarrhea initially improves, but the patient subsequently develops new-onset or worsening diarrhea after CDI treatment is completed, the recommendation is to submit a stool sample for CDI testing to evaluate for recurrent CDI. However, in the absence of new-onset or worsening diarrhea, repeat testing for CDI is not recommended to avoid the detection of asymptomatic $\mathrm{C}$ difficile colonization (see "Colonization vs infection," earlier).

\section{TREATMENT OF RECURRENT CDI}

C difficile infection is thought to primarily result from disruption of colonic microbiota
Stop

the inciting antibiotic

as soon as possible to avoid increasing the risk of recurrent CDI 


\section{C difficile infection}

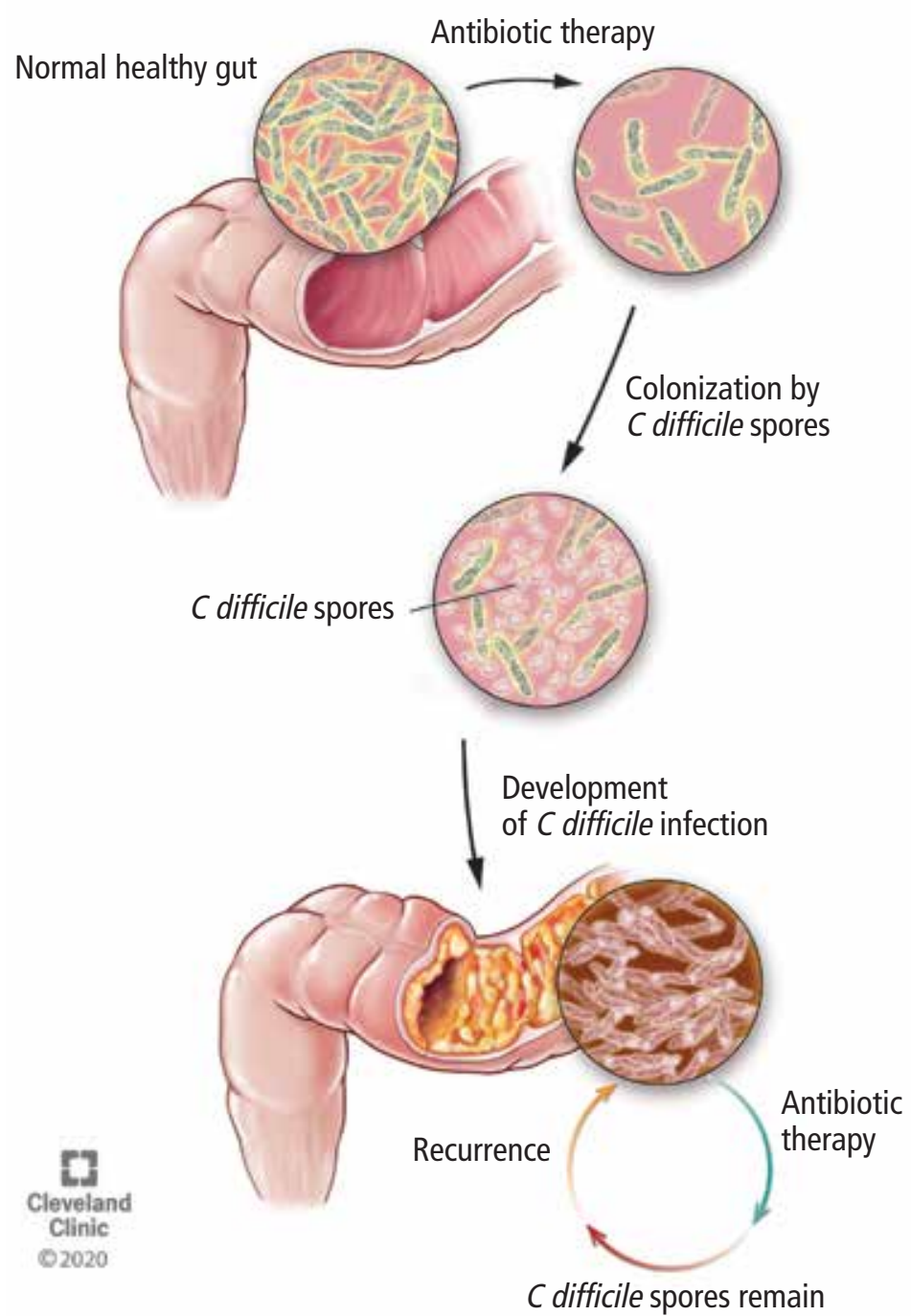

Figure 1. Antibiotic therapy can paradoxically lead to recurrent Clostridioides difficile infection by disrupting the normal colonic microbiota.

(Figure 1). Therefore, the primary goal of therapy for recurrent CDI is to allow the normal colonic microbiota to restore itself. ${ }^{11,12}$ Contributing to the difficulty of treating recurrent $\mathrm{CDI}$ is the ability of $\mathrm{C}$ difficile to transform from a vegetative gram-positive bacillus form, which is susceptible to killing by anti-C difficile therapy, to a spore form (Figure 2) that is resistant to anti-C difficile therapy and most other measures except hypochlorite-based solutions (ie, bleach). Antitoxin immune response may also be a factor in recurrent CDI. ${ }^{12}$

Treatment of recurrent CDI, according to the IDSA and SHEA guidelines, ${ }^{2}$ is based on the episode number of CDI, with nonsevere disease and severe disease being treated similarly.

A first recurrence (ie, a second episode), whether severe or nonsevere, has 3 options:

- Vancomycin, $125 \mathrm{mg}$ orally 4 times a day for 10 days (if metronidazole was used for the initial episode)

- Vancomycin in a tapered and pulsed regimen (rather than a second standard 10. day vancomycin course), such as $125 \mathrm{mg}$ orally 4 times per day for 10 to 14 days, then twice a day for 7 days, then once a day for 7 days, then every 2 or 3 days for 2 to 8 weeks

- Fidaxomicin 200 mg orally 2 times per day for 10 days (if vancomycin was used for the initial episode).

All 3 options carry weak recommendations, the first 2 based on low-quality evidence and the third based on moderate-quality evidence.

Oral vancomycin may be administered as a tapered and pulsed regimen. Tapering entails decreasing the dosage stepwise over a period of time to allow the normal colonic microbiota to restore itself. The pulsed regimen at the end of therapy entails dosing vancomycin every 2 to 3 days over a period of time to allow the treatment-resistant spore forms of $\mathrm{C}$ difficile to convert to the vegetative forms that are susceptible to killing by oral vancomycin.

A second recurrence, whether severe or nonsevere, has 4 options:

- Vancomycin in a tapered and pulsed regimen

- Vancomycin $125 \mathrm{mg} 4$ times a day by mouth for 10 days followed by rifaximin for 20 days

- Fidaxomicin $200 \mathrm{mg}$ orally 2 times a day for 10 days

- Fecal microbiota transplantation (more about this below).

Although the first 3 options carry weak recommendations based on low levels of evidence, fecal microbiota transplantation carries a strong recommendation based on a moderate level of evidence. ${ }^{2}$

\section{FIDAXOMICIN}

Fidaxomicin is a macrocyclic antibiotic that inhibits RNA synthesis. $\mathrm{C}$ difficile resistance or reduced susceptibility to fidaxomicin is rare, 
and it has no cross-resistance with rifamycin antibiotics. Like vancomycin, it is poorly absorbed. It exerts its activity in the gastrointestinal tract and has high fecal concentrations. ${ }^{13,14}$

Fidaxomicin is active against gram-positive anaerobes such as $\mathrm{C}$ difficile and Peptostreptococcus species, with variable activity against aerobic gram-positive cocci such as viridans streptococci and enterococci. However, it is less active against other anaerobic gram-positive bacilli such as Lactobacillus species, poorly active against anaerobic gram-negative bacilli (eg, Bacteroides species), and resistant to some Clostridium species (eg, C clostridioforme, $\mathrm{C}$ innocuum) that are key components of the normal colonic microbiota. ${ }^{13,14}$ Therefore, fidaxomicin should have a relatively lower impact on the normal colonic microbiota than therapies such as oral vancomycin.

But does it decrease the risk of recurrent CDI? In 2 randomized double-blind clinical trials comparing vancomycin with fidaxomicin,, 910 the clinical cure rates, defined as resolution of diarrhea 2 days after completing therapy, were similar. However, significantly fewer fidaxomicin-treated patients developed recurrent CDI. Louie and colleagues ${ }^{9}$ reported that $15.4 \%$ of patients developed recurrent CDI within 4 weeks of stopping fidaxomicin compared with $25.3 \%$ of patients who were treated with oral vancomycin $(P=.005$; absolute risk reduction $=9.9 \%$; number needed to treat $[\mathrm{NNT}]=10$; relative risk reduction $=39.1 \%)$. Cornely and colleagues ${ }^{10}$ reported similar results, with $12.7 \%$ of fidaxomicintreated patients developing recurrent CDI within 4 weeks compared with $26.9 \%$ of vancomycin-treated patients $(P=.0002$; absolute risk reduction $=14.2 \%$; NNT $=7$; relative risk reduction $52.7 \%$ ). Recurrent CDI was similar in the fidaxomicin and vancomycin groups among patients with the NAP1/BI/027 strain of $\mathrm{C}$ difficile (ie, North American pulsed-field gel electrophoresis type 1 , restriction endonuclease analysis pattern $\mathrm{BI}, \mathrm{PCR}$ ribotype designation 027), an epidemic strain of $\mathrm{C}$ difficile which emerged in the early 2000s. ${ }^{8}$ In contrast, recurrent CDI was lower with fidaxomicin compared with vancomycin among patients with non-NAP1/BI/027 strains of C difficile $(7.8 \%$ vs $25.5 \%$, respectively, in Louie et $\mathrm{al}^{9}$ and $9.2 \%$ vs $27.4 \%$ in Cornely et $\mathrm{al}^{10}$ ).

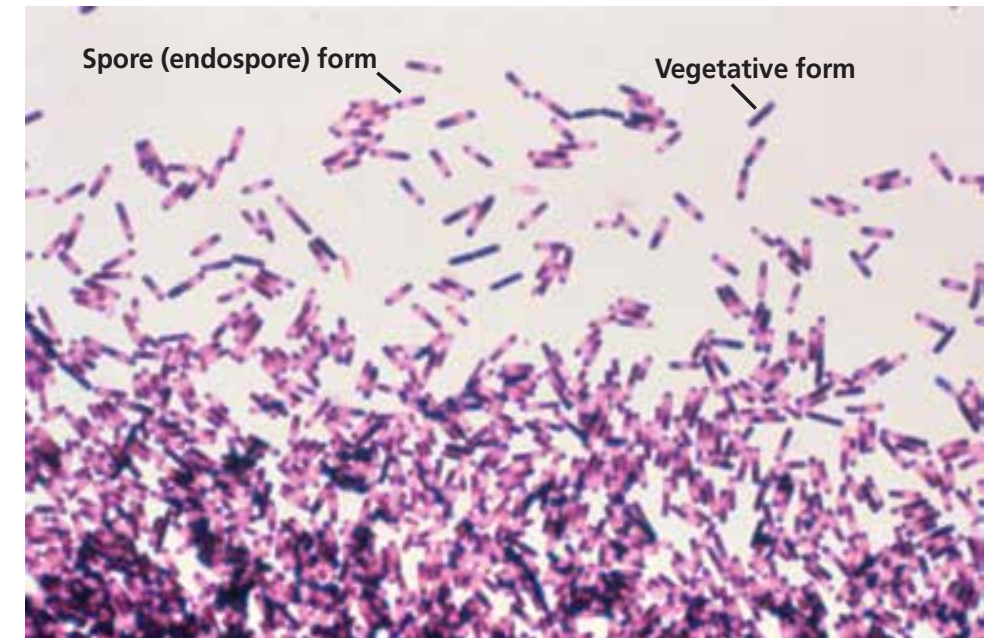

Figure 2. Photomicrograph showing the presence of Clostridioides difficile bacteria, many of which have assumed an endospore morphology. This is from a blood agar impression smear incubated for 72 hours anaerobically.

From the US Centers for Disease Control and Prevention, Dr. Gilda Jones; Public Domain; Available at: https://phil.cdc.gov/phil/details.asp?pid=3876

The NAP1/BI/027 strain of C difficile was the most prevalent strain of $\mathrm{C}$ difficile in the United States as far back as $2013 .{ }^{4}$ However, the prevalence of this strain has decreased over time both in healthcare-associated $\mathrm{C}$ difficile isolates (from $21 \%$ in 2012 to $15 \%$ in 2017) and in community-associated $\mathrm{C}$ difficile isolates (from $17 \%$ in 2012 to $6 \%$ in 2017), with the NAP1/BI/027 strain still being the most prevalent healthcare-associated strain in 2017, but no longer the most prevalent community-associated strain. ${ }^{4}$

Fidaxomicin is expensive, costing up to $\$ 4,500$ for a 10 -day course. ${ }^{15}$ In my experience, more insurance plans are covering fidaxomicin, but it is advised that before prescribing fidaxomicin, clinicians need to check whether the plan requires prior authorization and whether the patient can afford the copay.

\section{BEZLOTOXUMAB, A NEWER AGENT}

Poor antitoxin immune response may also play a role in recurrent CDI. ${ }^{11}$ Bezlotoxumab, an immunotherapeutic agent, received Food and Drug Administration (FDA) approval for prevention of recurrent CDI in October 2016. Efficacy was based on a large clinical trialMODIFY I and II - that enrolled 2,655 adults with primary or recurrent CDI. ${ }^{16}$ All were re-
Fidaxomicin is expensive and may require prior authorization 
ceiving standard-of-care anti-C difficile therapy. Investigators randomized the participants to either bezlotoxumab alone, actoxumab plus bezlotoxumab, or placebo. Actoxumab alone was given in MODIFY I but was discontinued after a planned interim analysis. Bezlotoxum$\mathrm{ab}$ is a human monoclonal antibody against $\mathrm{C}$ difficile toxin B. Actoxumab is a human monoclonal antibody against $\mathrm{C}$ difficile toxin A. The primary end point was recurrent CDI during 12 weeks of follow-up, defined as a new episode of CDI after the initial clinical cure.

Results showed similar initial cure rates in the pooled data set (ie, MODIFY I + II) with bezlotoxumab (80\%) and placebo (80\%); however, actoxumab plus bezlotoxumab had a significantly lower initial cure rate $(73 \%)$ than either placebo $(P=.0014)$ or bezlotoxumab alone $(P$ $=.0021)$. The initial cure rate with actoxumab alone (in MODIFY I) (73\%) was also significantly lower than placebo $(83 \% ; P=.0028)$.

Sustained cure rates (without recurrent infection) at 12 weeks in the pooled data set were higher with bezlotoxumab (64\%) than placebo (54\%; $P=.0001)$. Actoxumab plus bezlotoxumab showed no difference in the sustained cure rate $(58 \%)$ compared with pla-

Bezlotoxumab was approved after the guidelines were written cebo $(P=.0851)$ and a lower sustained cure rate compared with bezlotoxumab alone $(P=$ .0273). Sustained cure with actoxumab alone (in MODIFY I) (47\%) was lower than with placebo $(55 \%)(P=.0449)$.

Recurrent CDI in the pooled data set was lower with bezlotoxumab (17\%) and actoxumab plus bezlotoxumab (15\%) than with placebo $(27 \% ; P<.0001)$. Recurrent CDI with actoxumab alone (in MODIFY I) (26\%) was not different than with placebo $(28 \% ; P=.6364)$.

In summary, bezlotoxumab plus standardof-care was more effective than standard-ofcare alone in reducing the rate of recurrent CDI. Of note, compared with fidaxomicin in the trials discussed above, bezlotoxumab showed a similar absolute risk reduction (10.1\%), number needed to treat (10), and relative risk reduction $(37.9 \%)$ of recurrent CDI. In contrast, actoxumab alone or in combination with bezlotoxumab was inferior to placebo for the initial cure of CDI, and adding actoxumab to bezlotoxumab did not improve the efficacy of bezlotoxumab in reducing the rate of recurrent CDI.
Cost is a concern. Bezlotoxumab is expensive, costing approximately $\$ 4,500$ per patient course..$^{15}$ An editorial accompanying the MODIFY I and II trials suggested that bezlotoxumab use may vary depending on an analysis of cost versus the decrease in CDI recurrence risk compared with other options. ${ }^{17}$ Cost-effectiveness analyses have since been performed with conflicting conclusions. One study financed by the manufacturer found bezlotoxumab plus standard care (metronidazole, vancomycin, or fidaxomicin) was costeffective versus placebo plus standard care for primary or recurrent CDI. ${ }^{18}$ In contrast, Lam et $\mathrm{a}^{15}$ conducted a cost-effectiveness analysis specifically in patients with a first recurrence of CDI and concluded that vancomycin was the most cost-effective regimen for treating a first CDI recurrence. Fidaxomicin had higher quality-adjusted life years but at a cost higher than what was considered cost effective. Lastly, bezlotoxumab plus vancomycin was associated with a higher cost than fidaxomicin alone with an incremental decrease in quality-adjusted life years. The 2018 IDSA-SHEA clinical practice guidelines for CDI noted that bezlotoxumab received FDA approval after the guidelines were written and will be covered in subsequent guideline updates. ${ }^{2}$

\section{FECAL MICROBIOTA TRANSPLANTATION}

As discussed above, CDI is thought to primarily result from disruption of the normal colonic microbiota and can recur with many bouts over months or years despite standard therapies. The normal colonic microbiota is composed of a large and diverse community of microbes that resist colonization by new microbes (eg, $\mathrm{C}$ difficile), resulting in "colonization resistance." ${ }^{19-21}$ Antibiotics kill normal colonic microbiota and impair "colonization resistance," and impairment may last up to 4 weeks or longer.

FMT is the reintroduction of normal colonic microbiota from donor feces (Figure 3). It can be administered by the upper route (eg, nasogastric or nasoduodenal tube, esophagogastroduodenoscopy, or capsules) or the lower route (eg, colonoscopy, sigmoidoscopy, or enema). The source of stool for FMT is through human donors, either from donors that are 
known to the patient (eg, spouse, partner, friend) or from a prescreened volunteer donor pool (ie, stool bank).

To provide guidance for FMT, including indications, donor choice, donor exclusion criteria, donor testing, recipient exclusion criteria, and a protocol for performing FMT, the American Gastroenterological Association convened an expert work group that published guidelines in $2011^{20}$ and updated them in 2015.22 Recommended indications for FMT for patients with recurrent or relapsing CDI include at least 3 episodes of mild to moderate $\mathrm{CDI}$ and failure of a 6- to 8-week vancomycin taper, or at least 2 episodes of severe CDI resulting in hospitalization and associated with significant morbidity.

The American College of Gastroenterology published guidelines in 2013 that recommended considering FMT after 3 CDI recurrences (ie, 4 episodes) and receipt of a pulsed vancomycin regimen..$^{23}$ Clinical practice guidelines from IDSA and SHEA from 2018 recommended offering FMT only after patients have been diagnosed with at least 3 $\mathrm{CDI}$ episodes (ie, at least 2 recurrences) treated with appropriate anti-C difficile therapy. ${ }^{2}$

FMT is not FDA-approved, but its use is subject to FDA regulation. Also, there was concern that applying investigational new drug requirements would make FMT unavailable for patients. In July 2013, the FDA issued a Guidance for Industry statement noting that published data suggest FMT may be an effective therapy for management of refractory $\mathrm{CDI}$, but that the FDA intends to exercise enforcement discretion regarding investigational new drug requirements for use of FMT for CDI not responsive to standard therapies. Practitioners who recommend FMT must obtain informed consent from patients, which includes a discussion of its investigational status and its potential risks. ${ }^{24}$

\section{Evidence supporting FMT efficacy}

Before 2013, most of the published data on FMT use to treat recurrent CDI were from case reports. In a systematic review by Drekonja et a ${ }^{25}$ that included 480 patients treated with FMT for recurrent CDI from 21 case-series studies, FMT had an overall $85 \%$ cure rate (defined as resolution of symptoms

\section{Fecal microbiota transplantation}

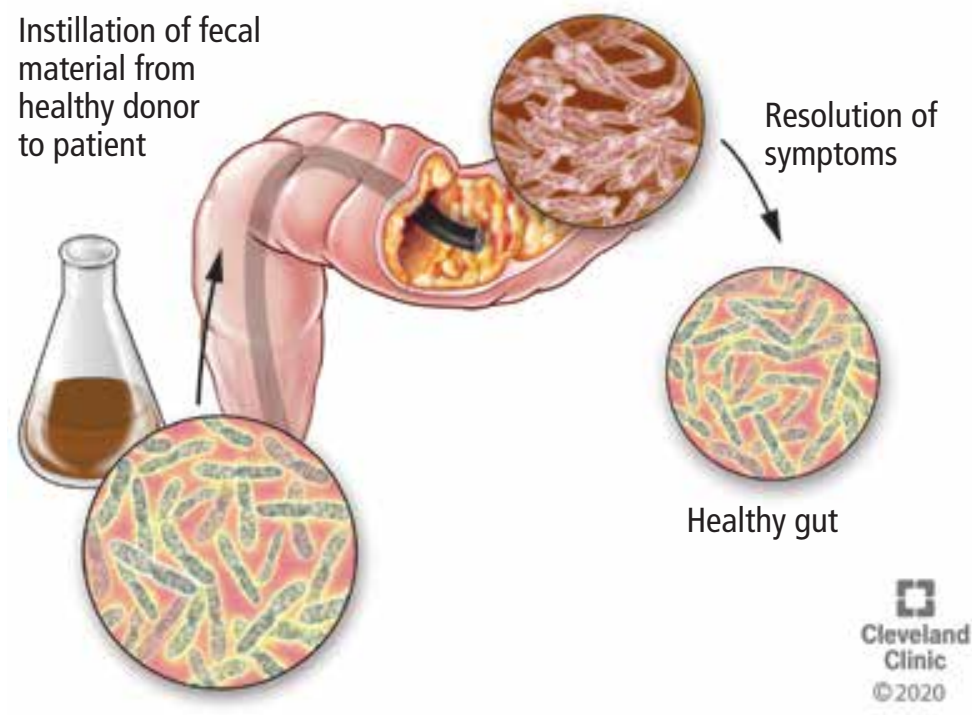

Figure 3. Fecal microbiota transplantation involves instilling fecal material from a healthy donor to restore the normal intestinal flora.

without recurrent CDI). The method of administration made a difference. Colonoscopic administration produced the highest resolution rate at $90 \%$, followed by enema administration at $78 \%$ and upper-gastrointestinal administration at $77 \%$. Although these cases show FMT has substantial success in recurrent CDI, it is relatively low-level evidence. In other reports, FMT administered orally via capsule has had variable success rates, ranging from $70 \%$ to $88 \% .26-29$

Clinical trials have shown similar efficacy of FMT for recurrent CDI. van Nood and colleagues ${ }^{30}$ compared FMT delivered by nasoduodenal tube vs oral vancomycin. The FMT recipients had $81 \%$ cure without recurrent CDI within 10 weeks after the first FMT and 94\% after 2 FMTs compared with 31\% cure for vancomycin $(P<.001)$. Cammarota and colleagues $^{31}$ compared FMT administered by colonoscopy vs oral vancomycin given in a pulsed regimen over at least 3 weeks. They reported cure rates without recurrent CDI within 10 weeks of $65 \%$ after the first FMT and 90\% after additional FMT procedures (ranging from 2 to 4 procedures) compared with $26 \%$ for vancomycin recipients $(P<.0001)$.

In 2016, in a randomized, double-blind, placebo-controlled clinical trial by Kelly et

\section{Fecal}

transplantation is not

FDA-approved, but it is subject to FDA regulation 
$\mathrm{al},{ }^{32}$ patients with 3 or more $\mathrm{CDI}$ recurrences received either FMT from a volunteer stool donor or a placebo composed of the patient's own stool. Both were administered by colonoscopy. All patients had received oral vancomycin for at least 10 days and continued it until 2 to 3 days before the FMT. The cure rate without recurrent CDI within 8 weeks was 91\% after first FMT and 100\% after second. Interestingly, the autologous FMT was successful $63 \%$ of the time, although the cure rate for donor FMT (91\%) was significantly higher $(P=.042)$. All patients who received an autologous FMT and developed recurrent CDI were then treated with a donor FMT, with a $100 \%$ success rate.

An open-label trial by Hota et $\mathrm{al}^{33}$ in 2017 compared FMT by enema vs oral vancomycin delivered in a tapered and pulsed regimen over 6 weeks. The cure rates without recurrent CDI within 4 months after FMT were surprisingly low-44\% for FMT and 58\% for vancomycin-and not consistent with the other published trials.

\section{FMT normalizes microbial diversity}

\section{The normal}

\section{colonic}

microbiota

is primarily

composed of

Firmicutes and

Bacteroidetes
The human microbiota, defined as the total collection of microorganisms within a community, is composed of an estimated 90 trillion microorganisms. Each body site (eg, colon, small bowel, oral cavity, skin) in the healthy human microbiota has a distinct microbial composition. The normal colonic microbiota is a highly diverse microbial community with 2 predominant bacterial phyla in healthy individuals, primarily composed of Firmicutes (approximately 50\%) and Bacteroidetes (approximately 30\%). ${ }^{21}$ Firmicutes include genera such as Clostridium, Lactobacillus, Streptococcus, Faecalibacterium, and Ruminococcus. Bacteroidetes are dominated by the genus Bacteroides. Less than 5\% of the colonic microbiota are composed of Proteobacteria, which includes Enterobacteriaceae such as Escherichia coli and Klebsiella species. ${ }^{21}$ The desirable mix is more Firmicutes and Bacteroidetes and fewer Proteobacteria.

Patients with recurrent CDI are known to have abnormal colonic microbiota, and FMT has been shown to normalize microbial diversity. In an FMT clinical trial by Kelly et $\mathrm{al},{ }^{32}$ fecal microbiome analysis (ie, total genes and gene products such as RNA and proteins produced by resident microbial communities) was performed at least 5 days before and 2 to 8 weeks after FMT. Before FMT, patient samples showed marked dysbiosis and lower diversity with more Proteobacteria and fewer Firmicutes and Bacteroidetes compared with donor samples. After FMT, patient samples showed normalization of the fecal microbiota.

The FMT clinical trial by van Nood and colleagues $^{30}$ found similar results. Before FMT, fecal microbial diversity was low. After FMT, microbial diversity was similar to that of donor stools with 2- to 4-fold more Bacteroidetes and clostridium clusters IV and XIVa and up to 100 -fold less Proteobacteria.

Similar changes in the fecal microbiome were seen in a clinical trial comparing vancomycin with fidaxomicin. ${ }^{14}$ Vancomycin led to a decrease in Bacteroides/Prevotella and Firmicutes group organisms, whereas fidaxomicin appeared to spare those groups.

\section{Adverse effects of FMT}

Adverse effects related to FMT may include the following ${ }^{34,35}$ :

- Transmission of infectious agents to the patient from the donor feces

- Complications from the FMT delivery procedure

- Long-term adverse effects related to the new colonic microbiota.

The human intestinal microbiota participates in a number of processes including maturation and continued education of the host immune response; regulation of intestinal endocrine functions and neurologic signaling; energy biogenesis; biosynthesis of vitamins and neurotransmitters; metabolism of bile salt; and reaction to or modification of certain drugs. ${ }^{36}$ The long-term impact of FMT on the development of illnesses such as metabolic syndrome and immune disorders is unknown. Screening donors for various diseases can help minimize these potential effects. ${ }^{34,35}$

Several reports of transmission of infectious agents from donor stool by FMT have been reported. In June 2019, the FDA published a safety alert on the transmission of extended-spectrum beta-lactamase (ESBL)producing $E$ coli after FMT in 2 immunocompromised patients. One patient died. 
The stools, which came from a single donor, were not tested for these organisms before the FMT. ${ }^{37}$ As a result, the FDA instituted requirements for stool donor screening questions regarding those with or at high risk for colonization with multidrug-resistant organisms (MDRO), and required that donor stool be tested for, at a minimum, ESBL-producing E coli, vancomycin-resistant enterococci (VRE), carbapenem-resistant Enterobacteriaceae, and methicillin-resistant Staphylococcus aureus. Additionally, the informed consent process for FMT should describe the risk of MDRO transmission and invasive infection and the measures implemented for donor screening and stool testing. ${ }^{38}$

Further information about these 2 patients was subsequently published. ${ }^{39}$ One of the patients had liver cirrhosis and was enrolled in a trial of FMT oral capsules to treat refractory hepatic encephalopathy. The other patient had undergone an allogeneic hematopoieticcell transplant and was enrolled in a trial of FMT oral capsules before and after the cell transplant. He developed neutropenia and fever on day 5 after stem cell infusion and was found to have ESBL-E coli bacteremia. He died 2 days later from severe sepsis.

In March 2020, the FDA published another safety alert regarding the suspected transmission of enteropathogenic E coli (EPEC) and Shigatoxin-producing E coli (STEC) from FMT products supplied by a stool bank and used to treat recurrent CDI. ${ }^{40}$ Two patients developed EPEC infection after receiving an FMT product prepared from stools from 2 different donors. Four patients developed STEC infection after receiving an FMT product prepared from a stool from a single donor. Four of the 6 patients required hospitalization, but none died. Additionally, there were 2 patients who died after receiving an FMT product manufactured from the donor associated with the 4 STEC infections. Both of these patients developed diarrhea after the FMT, but their stools were not tested for STEC. For one of the patients who died, the stool used to manufacture the FMT product was positive for STEC, but it is not known if STEC infection contributed to the patient's death. For the other patient who died, the stool used to manufacture the FMT product administered was negative for STEC, and the FDA did not suspect that the STEC was transmitted by this FMT product to this patient. ${ }^{41}$ In April 2020, the FDA recommended additional protections for FMT use, including testing donor stools by nucleic acid amplification tests for EPEC and STEC and excluding any stools testing positive. ${ }^{42}$

\section{PROBIOTICS}

Probiotics are preparations of viable microorganisms consumed by the patient. Studies have been conducted, but there are insufficient data to recommend probiotics for primary prevention of CDI.

The rationale for this conclusion is based on a Cochrane Review published in 2017 that evaluated the efficacy of probiotics for preventing $\mathrm{C}$ difficile-associated diarrhea (CDAD). ${ }^{43}$ Note that $\mathrm{C}$ difficile infection (CDI) was previously referred to as CDAD and there are clinical trials published using both terms. The CDI term was introduced in guidelines from IDSA and SHEA in 2010, ${ }^{2}$ and from the American College of Gastroenterology in $2013,^{23}$ and publications began using it. The authors of the Cochrane Review ${ }^{43}$ separated CDAD and CDI in their outcome groups and found that probiotics were effective only in preventing CDAD in patients whose baseline CDAD risk was greater than $5 \%(\mathrm{~N}=2,454$ in 13 trials; moderate certainty evidence). If the baseline CDAD risk was $0 \%$ to $2 \%(\mathrm{~N}=$ 5,845 patients in 15 trials; moderate certainty evidence) or $3 \%$ to $5 \%(\mathrm{~N}=373$ in 3 trials; low certainty evidence), probiotics had no effect on CDAD rates. Probiotics were also not effective in preventing $\mathrm{CDI}(\mathrm{N}=1,214$ in 15 trials; moderate certainty evidence).

Based on these meta-analysis findings and that typical CDI incidence rates are about 3\% or less, even during outbreaks, in hospitalized patients age 65 or older on antibiotics with a length of stay greater than 2 days, ${ }^{2}$ routine use of probiotics for inpatients on antibiotics for primary prevention of CDI is not recommended. 2,23

\section{PRIMARY AND SECONDARY CDI PROPHYLAXIS}

For patients who are on antibiotics to treat an infection other than CDI (eg, pneumonia),

\section{Routine use of probiotics for inpatients on antibiotics for primary prevention of CDI is not} recommended 
during or shortly after CDI treatment, the IDSA and SHEA CDI guidelines do not recommend extending the length of $\mathrm{CDI}$ treatment beyond the recommended duration or restarting CDI treatment shortly after completion of CDI therapy (ie, "secondary" CDI prophylaxis), due to insufficient data. ${ }^{2}$ The authors suggest that if a decision is made to institute secondary CDI prophylaxis, practitioners should consider low doses of vancomycin (eg, $125 \mathrm{mg}$ once daily) or fidaxomicin (eg, $200 \mathrm{mg}$ once daily) while the patient is on systemic antibiotics. ${ }^{2}$

For patients considered at high risk for developing CDI but who do not have active or recent CDI, administration of "primary" CDI prophylaxis (ie, administering anti-C difficile therapy to prevent $\mathrm{CDI}$ ) is not recommended. In a randomized nonblinded trial comparing vancomycin (125 mg once daily) in patients receiving systemic antibiotics versus no prophylaxis, there was a lower risk of developing hospital-onset CDI ( $0 \%$ vs $12 \%$, respectively; $P=.03$ ) with vancomycin prophylaxis; however, an analysis of the development of CDI after hospital discharge was limited by loss of follow-up. ${ }^{44}$ In a randomized double-blind placebo-controlled trial in patients undergoing

Patient rooms and environmental surfaces should be disinfected

with a hypochlorite solution hematopoietic stem cell transplant, fidaxomicin (200 mg once daily) treatment was not different from placebo for the primary composite end point of "prophylaxis failure" (28.6\% vs $30.8 \%$ with placebo, $P=.278) .{ }^{45}$ In a prespecified sensitivity analysis restricted to confirmed CDAD independent of missing data, CDAD was lower with fidaxomicin compared with placebo ( $4.3 \%$ vs $10.7 \%, P=.0014$ ); however, only $64 \%$ of subjects in each treatment group completed study treatment and follow-up. ${ }^{45}$

One of the principal concerns with CDI prophylaxis is that anti-C difficile therapies disrupt the normal colonic microbiota. Whether this affects CDI recurrence in the long term or increases the risk of colonization with multidrug-resistant bacteria is unknown. Vancomycin with a prolonged taper was shown in an animal model study to persistently disrupt the colonic microbiota, including a significant decrease in Firmicutes and increase in Proteobacteria, as well as to decrease colonization resistance to $\mathrm{C}$ difficile and vancomycinresistant enterococci. ${ }^{46}$ Fidaxomicin did not lead to disruption in colonization resistance.
In another animal model study, vancomycin markedly disrupted the microbiota and led to prolonged loss of colonization resistance to C difficile, vancomycin-resistant enterococci, carbapenem-resistant Klebsiella pneumoniae, and $E$ coli. Metronidazole had a more transient effect than vancomycin. ${ }^{47}$

\section{Strategies for CDI prevention}

There are 2 core strategies for preventing CDI: antibiotic stewardship, by implementing a program to optimize the use of antibiotics and minimize disruption of normal colonic microbiota, and infection prevention, by adhering to practices that block the spread and acquisition of the $\mathrm{C}$ difficile organism.

\section{Optimize use of antibiotics}

- Use antibiotics only when needed.

- Use narrow-spectrum antibiotics if possible. If the patient does not have a documented infection and lacks signs of sepsis, and if deemed appropriate by the treating clinician, consider waiting for culture testing results and then target the organism.

- Change from a broad-spectrum to a narrowspectrum antibiotic as soon as possible.

- Use the shortest possible treatment duration. The risk of developing CDI increases with longer antibiotic durations, with patients receiving more than 7 days of antibiotics having the highest risk. ${ }^{48}$

\section{Block the spread and acquisition of the $\boldsymbol{C}$ difficile organism}

$\mathrm{C}$ difficile is an obligate anaerobe able to survive and spread in the environment by conversion to spore form (Figure 2). Of note, C difficile is not part of normal colonic microbiota. C difficile spores can be transmitted from colonized patients to other patients either by healthcare workers (eg, on the hands) or from contaminated hospital environmental surfaces or equipment. These spores may then be ingested by noncolonized patients, survive exposure to the acidic environment of the stomach, and colonize the colonic lumen.

In a study published in 1989, McFarland et $\mathrm{al}^{49}$ found that of 399 patients admitted to a medical ward with negative admission $\mathrm{C}$ difficile rectal swab cultures, 83 (21\%) acquired $\mathrm{C}$ difficile during their hospitalization. The patients were tested for $\mathrm{C}$ difficile every 3 days. 
In addition, environmental cultures were collected during the study, and 62 of 216 (29\%) samples were positive. These included the bedrail, commode, floor, call button, windowsill, toilet, dialysis machine, sink, slipper bottoms, and nasogastric alimentation preparation. The frequency of positive environmental cultures was greater for patients with diarrhea (49\%) than for asymptomatic carriers (29\%). Further, C difficile cultures were taken from the hands of healthcare workers before and after interaction with patients whose cultures were positive, and 20 of 34 (59\%) hand cultures were positive for $\mathrm{C}$ difficile. ${ }^{49}$

Their findings emphasize the importance of blocking the spread of $\mathrm{C}$ difficile by physically washing away the spores with soap and water and by wearing gloves and gowns to prevent contact with the spores. Moreover, patient rooms and environmental surfaces should be disinfected with a hypochlorite-based solution (ie, bleach). This recommendation includes disinfection of equipment, such as blood pressure cuffs, thermometers, stethoscopes, and pen lights. If disposable equipment is not an option, then confine equipment to a single patient with CDI. Of course, all equipment shared between patients should be cleaned and disinfected between uses.

Other strategies to prevent $\mathrm{C}$ difficile include placing infected patients in a private room and using contact precautions while waiting for culture results. Encourage patients to wash their hands, especially before eating, as well as to shower or bed bathe to reduce the burden of spores on their skin.

\section{REFERENCES}

1. Lawson PA, Citron DM, Tyrrell KL, Finegold SM. Reclassification of Clostridium difficile as Clostridioides difficile (Hall and O'Toole 1935) Prévot 1938. Anaerobe 2016; 40:95-99. doi:10.1016/.anaerobe.2016.06.008

2. McDonald LC, Gerding DN, Johnson S, et al. Clinical practice guidelines for Clostridium difficile infection in adults and children: 2017 update by the Infectious Diseases Society of America (IDSA) and Society for Healthcare Epidemiology of America (SHEA). Clin Infect Dis 2018; 66(7):987-994. doi:10.1093/cid/ciy149

3. Lessa FC, Mu Y, Bamberg WM, et al. Burden of Clostridium difficile infection in the United States. N Engl J Med 2015; 372(9):825-834. doi:10.1056/NEJMoa1408913

4. Guh AY, Mu Y, Winston LG, et al. Trends in U.S. burden of Clostridioides difficile infection and outcomes. N Engl J Med 2020; 382(14):1320-1330. doi:10.1056/NEJMoa1910215

5. Bagdasarian N, Rao K, Malani PN. Diagnosis and treatment of Clostridium difficile in adults: a systematic review. JAMA 2015; 313(4):398-408. doi:10.1001/jama.2014.17103

6. Sethi AK, Al-Nassir WN, Nerandzic MM, Bobulsky GS, Donskey C. Persis-

\section{TAKE-HOME POINTS}

- Submit stool specimens for CDI testing only from patients with unexplained or new onset of at least 3 unformed stools in 24 hours.

- Do not treat patients who have asymptomatic $\mathrm{C}$ difficile carriage (ie, colonization); therapy has not been shown to be beneficial and can prolong $\mathrm{C}$ difficile colonization.

- For patients with CDI, stop the inciting antibiotic as soon as possible.

- For the first CDI episode, use vancomycin or fidaxomicin; metronidazole is only used as a third-line agent for nonsevere disease.

- For the second CDI episode, use vancomycin, vancomycin tapered and pulsed, or fidaxomicin.

- For a third CDI episode or more, use vancomycin tapered and pulsed, or vancomycin then rifaximin, or fidaxomicin, or fecal microbiota transplant.

- Fecal microbiota transplantation is associated with resolution of recurrent CDI, but its role in initial CDI episodes and fulminant CDI is not established.

- If considering bezlotoxumab therapy, assess the cost vs the recurrence risk compared with other options.

- To prevent CDI, optimize antibiotic use to minimize disruption of normal colonic microbiota, and physically block the spread and acquisition of the $\mathrm{C}$ difficile organism.

tence of skin contamination and environmental shedding of Clostridium difficile during and after treatment of $C$. difficile infection. Infect Control Hosp Epidemiol 2010; 31(1):21-27. doi:10.1086/649016

7. Johnson S, Homann SR, Bettin KM, et al. Treatment of asymptomatic Clostridium difficile carriers (fecal excretors) with vancomycin or metronidazole. a randomized, placebo-controlled trial. Ann Intern Med 1992; 117(4):297302. doi:10.7326/0003-4819-117-4-297

8. Cohen SH, Gerding DN, Johnson S, et al. Clinical practice guidelines for Clostridium difficile infection in adults: 2010 update by the Society for Healthcare Epidemiology of America (SHEA) and the Infectious Diseases Society of America (IDSA). Infect Control Hosp Epidemiol 2010; 31(5):431-455. doi:10.1086/651706

9. Louie TJ, Miller MA, Mullane KM, et al; OPT-80-003 Clinical Study Group. Fidaxomicin versus vancomycin for Clostridium difficile infection. N Engl J Med 2011; 364(5):422-431. doi:10.1056/NEJMoa0910812

10. Cornely OA, Crook DW, Esposito R, et al; OPT-80-004 Clinical Study Group. Fidaxomicin versus vancomycin for infection with Clostridium difficile in Europe, Canada, and the USA: a double-blind, non-inferiority, randomised controlled trial. Lancet Infect Dis 2012; 12(4):281-289. 
doi:10.1016/\$1473-3099(11)70374-7

11. Kelly CP, LaMont JT. Clostridium difficile - more difficult than ever. N Engl J Med 2008; 359(18):1932-1940. doi:10.1056/NEJMra0707500

12. Kelly CP. Can we identify patients at high risk of recurrent Clostridium difficile infection? Clin Microbiol Infect 2012; 18(suppl 6):21-27. doi:10.1111/1469-0691.12046

13. Kuijper EJ, Bauer MP. Fidaxomicin. In: Grayson ML. Kucer's The Use of Antibiotics. 7th ed. Boca Raton, FL: Taylor \& Francis Group; 2018.

14. Louie TJ, Cannon K, Byrne B, et al. Fidaxomicin preserves the intestinal microbiome during and after treatment of Clostridium difficile infection (CDI) and reduces both toxin reexpression and recurrence of CDI. Clin Infect Dis 2012; 55(suppl 2):S132-S142. doi:10.1093/cid/cis338

15. Lam SW, Neuner EA, Fraser TG, Delgado D, Chalfin DB. Cost-effectiveness of three different strategies for the treatment of first recurrent Clostridium difficile infection diagnosed in a community setting. Infect Control Hosp Epidemiol 2018; 39(8):924-930. doi:10.1017/ice.2018.139

16. Wilcox MH, Gerding DN, Poxton IR, et al; MODIFY I and MODIFY II Investigators. Bezlotoxumab for prevention of recurrent Clostridium difficile infection. N Engl J Med 2017; 376(4):305-317. doi:10.1056/NEJMoa1602615

17. Bartlett JG. Bezlotoxumab - a new agent for Clostridium difficile infection N Engl J Med 2017; 376(4):381-382. doi:10.1056/NEJMe1614726

18. Prabhu VS, Dubberke ER, Dorr MB, et al. Cost-effectiveness of bezlotoxumab compared with placebo for the prevention of recurrent Clostridium difficile infection. Clin Infect Dis 2018; 66(3):355-362. doi:10.1093/cid/cix809

19. $\mathrm{Na} \mathrm{X,} \mathrm{Kelly} \mathrm{C.} \mathrm{Probiotics} \mathrm{in} \mathrm{Clostridium} \mathrm{difficile} \mathrm{infection.} \mathrm{J} \mathrm{Clin} \mathrm{Gastroen-}$ terol 2011; 45(suppl):S154-S158. doi:10.1097/MCG0b013e31822ec787

20. Bakken JS, Borody T, Brandt U, et al; for the Fecal Microbiota Transplantation Workgroup. Treating Clostridium difficile infection with fecal microbiota transplantation. Clin Gastroenterol Hepatol 2011; 9(12):1044-1049. doi:10.1016/j.cgh.2011.08.014

21. Aagaard K, Luna RA, Versalovic J. The human microbiome of local body sites and their unique biology. In: Bennett JE, Dolin R, Blaser MJ, eds. Mandell, Douglas, and Bennett's Principles and Practice of Infectious Diseases, 8th ed. Philadelphia, PA: Elsevier Inc; 2015.

22. Kelly CR, Kahn S, Kashyap P, et al. Update on fecal microbiota transplantation 2015: indications, methodologies, mechanisms, and outlook. Gastroenterology 2015; 149(1):223-237. doi:10.1053/j.gastro.2015.05.008

23. Surawicz CM, Brandt U, Binion DG, et al. Guidelines for diagnosis, treatment, and prevention of Clostridium difficile infections. Am J Gastroenterol 2013; 108(4):478-498. doi:10.1038/ajg.2013.4

24. US Food and Drug Administration. Guidance for industry: enforcement policy regarding investigational new drug requirements for use of fecal microbiota for transplantation to treat Clostridium difficile infection not responsive to standard therapies. July 2013. Accessed May 5, 2020. https:// www.fda.gov/media/86440/download

25. Drekonja D, Reich J, Gezahegn S, et al. Fecal microbiota transplantation for Clostridium difficile infection: a systematic review. Ann Intern Med 2015; 162(9):630-638. doi:10.7326/M14-2693

26. Youngster I, Russell GH, Pindar C, Ziv-Baran T, Sauk J, Hohmann EL. Oral, capsulized, frozen fecal microbiota transplantation for relapsing Clostridium difficile infection. JAMA 2014; 312(17):1772-1778. doi:10.1001/jama.2014.13875

27. Youngster I, Mahabamunuge J, Systrom HK, et al. Oral, frozen fecal microbiota transplant (FMT) capsules for recurrent Clostridium difficile infection. BMC Med 2016;14(1):134. doi:10.1186/s12916-016-0680-9

28. Staley C, Hamilton MJ, Vaughn BP, et al. Successful resolution of recurrent Clostridium difficile infection using freeze-dried, encapsulated fecal microbiota; pragmatic cohort study. Am J Gastroenterol 2017; 112(6):940-947. doi:10.1038/ajg.2017.6

29. Jiang ZD, Jenq RR, Ajami NJ, et al. Safety and preliminary efficacy of orally administered lyophilized fecal microbiota product compared with frozen product given by enema for recurrent Clostridium difficile infection: a randomized clinical trial. PLoS One 2018; 13(11):e0205064. doi:10.1371/journal.pone.0205064
30. van Nood E, Vrieze A, Nieuwdorp M, et al. Duodenal infusion of donor feces for recurrent Clostridium difficile. N Engl J Med 2013; 368(5):407-415. doi:10.1056/NEJMoa1205037

31. Cammarota G, Masucci L, laniro G, et al. Randomised clinical trial: faecal microbiota transplantation by colonoscopy vs. vancomycin for the treatment of recurrent Clostridium difficile infection. Aliment Pharmacol Ther 2015; 41(9):835-843. doi:10.1111/apt.13144

32. Kelly CR, Khoruts A, Staley C, et al. Effect of fecal microbiota transplantation on recurrence in multiply recurrent Clostridium difficile infection: a randomized trial. Ann Intern Med 2016; 165(9):609-616. doi:10.7326/M16-0271

33. Hota SS, Sales V, Tomlinson G, et al. Oral vancomycin followed by fecal transplantation versus tapering oral vancomycin treatment for recurrent Clostridium difficile infection: an open-label, randomized controlled trial. Clin Infect Dis 2017; 64(3):265-271. doi:10.1093/cid/ciw731

34. Brandt $\sqcup$, Aroniadis OC, Mellow M, et al. Long-term follow-up of colonoscopic fecal microbiota transplant for recurrent Clostridium difficile infection. Am J Gastroenterol 2012; 107(7):1079-1087. doi:10.1038/ajg.2012.60

35. Orenstein R, Griesbach CL, DiBaise JK. Moving fecal microbiota transplantation into the mainstream. Nutr Clin Pract 2013;28(5):589-598. doi:10.1177/0884533613497516

36. Lynch SV, Pedersen O. The human intestinal microbiome in health and disease. N Engl J Med 2016; 375(24):2369-2379. doi:10.1056/NEJMra1600266

37. US Food and Drug Administration. Important safety alert regarding use of fecal microbiota for transplantation and risk of serious adverse reactions due to transmission of multi-drug resistant organisms. June 13, 2019. https://www.fda.gov/vaccines-blood-biologics/safety-availability-biologics/ information-pertaining-additional-safety-protections-regarding-use-fecalmicrobiota-transplantation

38. US Food and Drug Administration. Information pertaining to additional safety protections regarding use of fecal microbiota for transplantation screening and testing of stool donors for multi-drug resistant organisms. June 18, 2019. https://www.fda.gov/vaccines-blood-biologics/safetyavailability-biologics/information-pertaining-additional-safety-protectionsregarding-use-fecal-microbiota-transplantation

39. DeFilipp Z, Bloom PP, Torres Soto M, et al. Drug-resistant $E$. coli bacteremia transmitted by fecal microbiota transplant. N Engl J Med 2019; 381(21):2043-2050. doi:10.1056/NEJMoa1910437

40. US Food and Drug Administration. Safety alert regarding use of fecal microbiota for transplantation and risk of serious adverse events likely fue to transmission of pathogenic organisms. March 12, 2020. Accessed May 5, 2020. https://www.fda.gov/vaccines-blood-biologics/safety-availabilitybiologics/safety-alert-regarding-use-fecal-microbiota-transplantation-andrisk-serious-adverse-events-likely

41. US Food and Drug Administration. Update to March 12, 2020 safety alert regarding use of fecal microbiota for transplantation and risk of serious adverse events likely due to transmission of pathogenic organisms. March 13, 2020. Accessed May 5, 2020. https://www.fda.gov/vaccines-blood-biologics/ safety-availability-biologics/update-march-12-2020-safety-alert-regardinguse-fecal-microbiota-transplantation-and-risk-serious

42. US Food and Drug Administration. Information pertaining to additional safety protections regarding use of fecal microbiota for transplantation - testing of stool donors for enteropathogenic Escherichia coli and shigatoxin-producing Escherichia coli. April 6, 2020. Accessed May 5, 2020. www.fda.gov/vaccines-blood-biologics/safety-availability-biologics/ information-pertaining-additional-safety-protections-regarding-use-fecalmicrobiota-transplantation-0

43. Goldenberg JZ, Yap C, Lytvyn L, et al. Probiotics for the prevention of Clostridium difficile-associated diarrhea in adults and children. Cochrane Database Syst Rev 2017; 12:CD006095. doi:10.1002/14651858.CD006095.pub4

44. Johnson SW, Brown SV, Priest DH. Effectiveness of oral vancomycin for prevention of healthcare facility-onset Clostridioides difficile infection in targeted patients during systemic antibiotic exposure. Clin Infect Dis 2019. doi:10.1093/cid/ciz966

45. Mullane KM, Winston DJ, Nooka A, et al. A randomized, placebo-controlled trial of fidaxomicin for prophylaxis of Clostridium difficile-associated 
diarrhea in adults undergoing hematopoietic stem cell transplantation. Clin Infect Dis 2019; 68(2):196-203.

doi:10.1093/cid/ciy484

46. Tomas ME, Mana TSC, Wilson BM, et al. Tapering courses of oral vancomycin induce persistent disruption of the microbiota that provide colonization resistance to Clostridium difficile and vancomycin-resistant enterococci in mice. Antimicrob Agents Chemother 2018; 62(5):e02237-17. doi:10.1128/AAC.02237-17

47. Lewis BB, Buffie CG, Carter RA, et al. Loss of microbiota-mediated colonization resistance to Clostridium difficile infection with oral vancomycin compared with metronidazole. J Infect Dis 2015; 212(10):1656-1665. doi:10.1093/infdis/jiv256

48. Dubberke ER, Reske KA, Yan Y, Olsen MA, McDonald LC, Fraser VJ. Clostridium difficile-associated disease in a setting of endemicity: identification of novel risk factors. Clin Infect Dis 2007; 45(12):1543-1549. doi: $10.1086 / 523582$

49. McFarland LV, Mulligan ME, Kwok RY, Stamm WE. Nosocomial acquisition of Clostridium difficile infection. N Engl J Med 1989; 320(4):204-210. doi:10.1056/NEJM198901263200402

Address: Constantine Tsigrelis, MD, Department of Infectious Disease, G21, Cleveland Clinic, 9500 Euclid Avenue, Cleveland, $\mathrm{OH} 44195$; tsigrec@ccf.org 